\title{
Generalized pseudo-Newtonian potential for studying accretion disk dynamics in off-equatorial planes around rotating black holes: Description of a vector potential
}

\author{
Shubhrangshu Ghosh ${ }^{1}$ and Banibrata Mukhopadhyay ${ }^{1}$
}

\begin{abstract}
We prescribe a pseudo-Newtonian vector potential for studying accretion disks around Kerr black holes. The potential is useful to study the inner properties of disk not confined to the equatorial plane where general relativistic effect is indispensable. Therefore, we incorporate the essential properties of the metric at the inner radii through the pseudo-Newtonian potential derived from the general Kerr spacetime. The potential, reproducing most of the salient features of the general-relativity, is valid for entire regime of Kerr parameter. It reproduces the last stable circular orbit exactly as that in the Kerr geometry. It also reproduces last bound orbit and energy at last stable circular orbit with a maximum error $\sim 7 \%$ and $\sim 15 \%$ respectively upto an orbital inclination $30^{\circ}$.
\end{abstract}

Subject headings: accretion, accretion disk — black hole physics — gravitation - relativity

\section{Introduction}

The standard model of the accretion disk proposed by Shakura \& Sunyaev (1973) explains the dynamics of a thin disk by the Newtonian potential, not taking into account of the essential general-relativistic effects of the black hole. The only notion it hires from general relativity (hereinafter GR) is that the inner most edge of the disk truncates at the last stable circular orbit of the Schwarzschild geometry. Complete general relativistic description of the thin Keplerian disk was developed by Novikov \& Thorne (1973). Owing to the complicated and cumbersome nature of underlying general relativistic equations, especially to study the full scale dynamics of the disk, it would be worthy enough to study in Newtonian framework using a modified Newtonian potential, namely pseudo-Newtonian potential

\footnotetext{
${ }^{1}$ Astronomy and Astrophysics Program, Department of Physics, Indian Institute of Science, Bangalore 560012, India; bm@physics.iisc.ernet.in
} 
(hereinafter PNP), having all the essence of a black hole geometry. Such a potential was first proposed by Paczyński \& Wiita (1980; hereinafter PW) for modeling a thick/thin accretion disk around a nonrotating black hole. The potential exactly reproduces marginally stable orbit $\left(r_{s}\right)$ and marginally bound orbit $\left(r_{b}\right)$ of that in full GR and the efficiency per unit mass at the last stable circular orbit $\left(E_{s}\right)$ agrees with at most $10 \%$ error. Nowak \& Wagoner (1991) and Mukhopadhyay \& Misra (2003) proposed other potentials for an accretion disk to describe epicyclic frequency which mimic most of the properties of the disk governed by the Schwarzschild and Kerr geometry respectively.

PNPs to describe the fluid dynamics of accretion disk around a rotating black hole in equatorial plane were also proposed by Chakrabarti \& Khanna (1992) and Artemova et al. (1996) which, however, were not valid for entire range of Kerr parameter. Later, a twodimensional potential was described by Mukhopadhyay (2002; hereinafter M02) which is valid for the entire range and reproduces $r_{s}$ exactly, $r_{b}$ with a maximum error $\sim 5 \%$ and $E_{s}$ with a maximum possible error $\sim 10 \%$ compared to that in the Kerr geometry. The more interesting fact, however, lies in the methodology adopted by M02 in deriving the potential which can be used to formulate the PNP for any metric according to the physics concern. Based on the procedure, several authors have developed PNPs at the equatorial plane for other metric, e.g Hartle-Thorne metric by Ghosh (2004), Kerr-Newman metric by Ivanov \& Prodanov (2005). We plan to follow the procedure outlined by M02 to derive a PNP for general Kerr geometry (particle motion not confined to the equatorial plane) which can be applied to study the generalized axisymmetric accretion disk dynamics. Naturally, the aim is to furnish a pseudo-Newtonian vector potential rather than a scalar potential with radial and vertical component in cylindrical coordinate system suitable for studying a thick accretion disk.

While some authors focus on to propose an ad-hoc and a mathematically simpler form of PNP, we derive it from the spacetime metric directly. Therefore, we do not need to worry about its final form strictly determined by our procedure for any spacetime metric unlike the other PNPs. Problem with an ad-hoc PNP is that there is no easy one to one correspondence between spacetime metric and PNP.

Most of the cosmic objects in our nature are rotating (see e.g. Iwasawa et al. 1996; Wilms et al. 2001; Miniutti et al. 2004; Miller et al. 2004; Genzel et al. 2003). Recently, it has also been argued (McClintock et al. 2006) that the source GRS 1915+105 is a rapidly rotating black hole with spin parameter $a>0.98$. Thus, the predictions of the inner disk properties will be incorrect if the pseudo-Newtonian modeling fails to include the spin parameter of the black hole. The potential proposed by M02 has already been used to study the hydrodynamics of the disk around Kerr black holes, which shows a dramatic 
change of the valid parameter space region; any shock location and other fluid properties change for different rotation parameters of the black hole (Mukhopadhyay 2003). Recently Chan et al. $(2005,2006)$ have used this potential for time dependent studies of accretion flow for twodimensional viscous hydrodynamic disk and for threedimensional self-gravitating magnetohydrodynamic disk around rotating black holes using spectral method.

Our aim in the present paper is to develop a potential for the general Kerr geometry which can be used to mimic the general relativistic effects of the spacetime, enabling us to suitably use it to study the accretion dynamics, especially of a thick disk with the inclusion of vertical outflow. Thus we goal for a generalized potential which is a function of $r$ and $\theta$ both and having two components, in radial and vertical direction, in cylindrical coordinate system, hence a vector potential. This is useful for both quasi-numerical analyzes and time dependent simulations, avoiding the use of complicated relativistic equations, yet capturing the essential physics of spacetime outside of a Kerr black hole. In early, some attempt was made to describe PNP in generalized Kerr geometry by, e.g., Dadhich (1985), Semerák \& Karas (1999), Chakrabarti \& Mondal (2006). The PNPs described in first two works, while aim to furnish frame-dragging effects in the Newtonian framework, do not reproduce marginally stable and bound circular orbits and specific energy close to the black hole and hence may not be suitable for the hydrodynamical analysis of a Keplerian accretion disk, particularly, when aim is to study inner disk properties. The third one is the modification of Chakrabarti \& Khanna (1992) potential to mimic approximately the general relativistic effects in accretion disks for $-1 \leq a \leq 0.8$. The potential is a prescription with which the authors maintain the ad-hoc-ness of PW potential and may be used to study accretion disk having particle orbits of small inclination angle at a certain parameter window. Our goal here, however, is to extend the procedure initiated by M02 which can be used to describe a PNP for the general Kerr spacetime; particle motion not confined to the equatorial plane. The potential given by M02 is more useful to study the dynamics of a vertically averaged thin disk at equatorial plane. Henceforth, we wish to derive a generalized potential applicable for off-equatorial planes following the procedure of M02 which reproduces exactly or in good agreement all the (inner) accretion disk properties (unlike the previous works) for a general case in Kerr geometry. This will be useful to study the thick accretion disk dynamics with the inclusion of vertical outflow and wind. The potential should reproduce those features of a rotating black hole geometry which have been reproduced by that of M02 at equatorial plane and that of PW for a nonrotating black hole. We formulate our PNP from the Kerr metric. As the metric is involved directly to our calculation, many of the features of Kerr geometry are inherent in our potential. The axisymmetric PNP we plan to establish here for a general Kerr metric is more useful for quasi-analytical studies and time dependent simulations of accretion and wind and related physics as that studied by Chan et al. $(2005,2006)$, Lipunov 
\& Gorbovskoy (2007), Shafee et al. (2007) using M02, without loss of any generality.

We arrange the paper in the following manner. In the next section, we present the basic equations and derive the PNP. In $\S 3$, we compare the various features of dynamics of accreting particles obtained by our potential with that in Kerr geometry. Finally $\S 4$ presents a discussion with summary.

\section{Formulation of the generalized pseudo-Newtonian potential}

Here we furnish the derivation of our said potential. It is condensed in three subsections, where we have elucidated the description of the potential starting from the Kerr metric.

\subsection{Integrals of motion of a test particle in the Kerr geometry}

The Kerr spacetime in the Boyer-Lindquist coordinate system in geometrized units $G=c=1$ is given by

$$
\begin{aligned}
d s^{2}= & -\left(1-\frac{2 M r}{\Sigma}\right) d t^{2}-\frac{4 a M r \sin ^{2} \theta}{\Sigma} d t d \phi+\frac{\Sigma}{\Delta} d r^{2} \\
& +\Sigma d \theta^{2}+\left(r^{2}+a^{2}+\frac{2 M r a^{2} \sin ^{2} \theta}{\Sigma}\right) \sin ^{2} \theta d \phi^{2},
\end{aligned}
$$

where $\Delta=r^{2}+a^{2}-2 M r, \Sigma=r^{2}+a^{2} \cos ^{2} \theta$ and $a=\frac{J}{M}$ which is specific angular momentum of the black hole named Kerr parameter. The Lagrangian density of the particle of mass $m$ in the Kerr spacetime is given by

$$
\begin{aligned}
2 \mathcal{L} & =-\left(1-\frac{2 M r}{\Sigma}\right) \dot{t}^{2}-\frac{4 a M r \sin ^{2} \theta}{\Sigma} \dot{t} \dot{\phi}+\frac{\Sigma}{\Delta} \dot{r}^{2} \\
& +\Sigma \dot{\theta}^{2}+\left(r^{2}+a^{2}+\frac{2 M r a^{2} \sin ^{2} \theta}{\Sigma}\right) \sin ^{2} \theta \dot{\phi}^{2},
\end{aligned}
$$

where over-dots denote the derivative with respect to proper-time $\tau$ along the curve. From the symmetries, we obtain two constants of motion corresponding to two ignorable coordinates $t$ and $\phi$ given by

$$
p_{t}=\frac{\partial \mathcal{L}}{\partial \dot{t}}=-\left(1-\frac{2 M r}{\Sigma}\right) \dot{t}-\frac{2 a M r \sin ^{2} \theta}{\Sigma} \dot{\phi}=\text { constant }=-E
$$

and

$$
p_{\phi}=\frac{\partial \mathcal{L}}{\partial \dot{\phi}}=-\frac{2 a M r \sin ^{2} \theta}{\Sigma} \dot{t}+\left(r^{2}+a^{2}+\frac{2 M r a^{2} \sin ^{2} \theta}{\Sigma}\right) \sin ^{2} \theta \dot{\phi}=\text { constant }=\lambda_{z},
$$


where $\lambda_{z}$ is the component of angular momentum of the orbiting particle along spin axis of the black hole (i.e. parallel to the symmetry axis). The Hamiltonian of the system can also be written as

$$
\mathcal{H}=\frac{1}{2} g^{\mu \nu} p_{\mu} p_{\nu}
$$

The Hamiltonian is itself a constant of motion and by usual setting $g_{\mu \nu} p^{\mu} p^{\nu}=-m^{2}$ for a particle with nonzero rest mass, we obtain the third constant of motion (integral of motion) as

$$
\mathcal{H}=\frac{1}{2} m^{2}=\mathcal{L}=\text { constant }
$$

The Lagrangian and the Hamiltonian of the geodesic with the above condition are exactly same. The three integrals of motion obtained above are sufficient to determine the geodesic equations under restrictive condition like the motion of a test particle confined to an equatorial plane $(\dot{\theta}=0, \theta=\pi / 2)$ (e.g. Shapiro \& Teukolsky 1983). To describe the motion of a particle for a generic case around the Kerr black hole, with dependency on $\theta$, a fourth integral of motion is needed which does not come from the symmetries of the metric. It was discovered by Carter (1968) that such an integral of motion can be obtained explicitly by separability of the Hamilton-Jacobi equation (hereinafter HJE) (also see Chandrasekhar 1983) given by

$$
Q=p_{\theta}^{2}+\cos ^{2} \theta\left[a^{2}\left(m^{2}-E^{2}\right)+\frac{\lambda_{z}^{2}}{\sin ^{2} \theta}\right]
$$

$Q$ is called the Carter constant (Carter 1968). The above equation together with eqns. (3) and (41) provides a complete set of integrals of motion for the geodesic of a test particle in the Kerr geometry where $Q, E$ and $\lambda_{z}$ are three constants motion. It can be seen from the above equation that for a particle always confined to the equatorial plane (i.e., $\dot{\theta}=0$ and $\theta=\pi / 2)$, the Carter constant $Q$ is zero. For $a=0, Q+\lambda_{z}^{2}=\lambda^{2}$ is square of the total angular momentum of a particle's orbital motion. It is to be noted that the information about $\theta$ and $\dot{\theta}$ for an orbital trajectory is consolidated into a single parameter $Q$, which is a conserved quantity. Now using eqns. (2), (3), (4), (6) and (7), we obtain the most general equations of motion governing the orbital trajectory of a test particle in the Kerr geometry (Bardeen 1972; Wilkins 1972) given by

$$
\begin{aligned}
& \Sigma \frac{d r}{d \tau}= \pm \sqrt{R} \\
& \Sigma \frac{d \theta}{d \tau}= \pm \sqrt{\Theta}
\end{aligned}
$$




$$
\begin{gathered}
\Sigma \frac{d \phi}{d \tau}=-a E+\frac{\lambda_{z}}{\sin ^{2} \theta}+\frac{a}{\Delta}\left[E\left(r^{2}+a^{2}\right)-\lambda_{z} a\right], \\
\Sigma \frac{d t}{d \tau}=-a^{2} E \sin ^{2} \theta+a \lambda_{z}+\frac{r^{2}+a^{2}}{\Delta}\left[E\left(r^{2}+a^{2}\right)-\lambda_{z} a\right],
\end{gathered}
$$

where

$$
\begin{gathered}
R=\left[E\left(r^{2}+a^{2}\right)-\lambda_{z} a\right]^{2}-\Delta\left[m^{2} r^{2}+\left(\lambda_{z}-a E\right)^{2}+Q\right], \\
\Theta=Q-\cos ^{2} \theta\left[a^{2}\left(m^{2}-E^{2}\right)+\frac{\lambda_{z}^{2}}{\sin ^{2} \theta}\right] .
\end{gathered}
$$

\subsection{Effective potentials and prescription of orbital inclination}

$R$ and $\Theta$ are in general called the effective potentials describing the particle motion in a generalized Kerr spacetime. For a particle moving perpetually at the equatorial plane, the only effective potential that governs the particle motion is $R$ with $Q$ equals to zero. In order to understand the generic orbit, both potentials should be accounted for. To have an insight of the nature of orbits in threedimensional Kerr geometry, we recall that for bound geodesics $(Q \geq 0)$ orbits either remain perpetually at the equatorial plane $(Q=0)$ or cross it repeatedly $(Q>0)$. More precisely, orbits with $Q>0$ (more general case), also called the off-equatorial orbits, are inclined to the equatorial orbital plane. Figure 1 shows that the orbital plane of an off-equatorial orbit is inclined at an angle $i$ to the equatorial plane, which also means that the orbital plane makes an inclination $i$ with the spin axis of the hole (symmetry axis). For a particular orbit, the inclination angle $i$ is a constant of motion. However, in general, the plane of an off-equatorial orbit precesses with an angular frequency $2 a / r^{3}$ called Lense-Thirring precession (Lense \& Thirring 1918). Owing to this fact, the plane of the orbit neither remains fixed, nor crosses the equatorial plane with constant inclination $i$, but rather at a different angle. Looking meticulously, we find that the information of entire $\theta$ motion (as for a particular off-equatorial orbit, $\theta$ has all arbitrary values and it changes constantly) is glued up into one single inclination parameter $i$. Thus the motion of the bound orbit in the Kerr geometry is now governed by four parameters $r, i, \phi$ and $t$.

As our intention is to derive a generalized PNP for particle orbits not confined to the equatorial plane, which itself is an approximate quantity, we restrict ourselves to an ideal case of circular orbits. In general, due to plane precession, the orbit never remains circular, but have eccentricity. The study of generic orbits (inclined and eccentric) for our particular case is deferred for future. The circular orbits satisfy $R=d R / d r=0$. Hence to analyze the orbital motion for circular case, the radial effective potential $R$ is necessary and sufficient. 
Our next objective is to describe the inclination parameter $i$ with known quantities. As stated earlier, the constant inclination angle $i$ (without considering plane precession) is the angle between the plane of the orbit and the spin axis of the hole. As $\lambda^{2}=Q+\lambda_{z}^{2}$ for $a=0$ stated in the preceding subsection, we identify in the Cartesian coordinate system $Q \sim \lambda_{x}^{2}+\lambda_{y}^{2}$ approximately. Hence $Q$ can be treated as the component of square of the total angular momentum in perpendicular to the symmetry axis or to the spin axis $\left(\lambda_{\perp}\right)$ of the black hole or precisely the component projected into the equatorial plane. However, even for a maximally spinning hole the error is found to be less than a few percent (Glampedakis et al. 2002). With this conjecture, the inclination parameter $i$ can be defined as (Ryan 1995; Shapiro 1996; Hughes 2000)

$$
\cos i=\frac{\lambda_{z}}{\sqrt{Q+\lambda_{z}^{2}}}
$$

Taking all these into account, our radial effective potential $R$ in eqn. (12) with further simplification eventually reduces to

$$
R=r\left(r^{3}+r a^{2}+2 M a^{2}\right) E^{2}-r(r-2 M) \lambda_{z}^{2}-4 a M r \lambda_{z} E-\Delta m^{2} r^{2}-\Delta \lambda_{z}^{2} \tan ^{2} i .
$$

Thus the effective potential is a function of $r$ and $i$ (which is a conserved quantity for a particular orbit). If we consider the effect of Lense-Thirring precession on the orbital plane, then we need to amend a minor correction with the above definition of inclination angle. Now if you specify for circular orbits, then the effective potential further reduces as

$V_{\mathrm{eff}}=\frac{2 a M \lambda_{z}+\left[\lambda_{z}^{2}\left\{r^{2}+\frac{\tan ^{2} i}{r}\left(r^{3}+r a^{2}+2 M a^{2}\right)\right\} \Delta+m^{2} r\left(r^{3}+r a^{2}+2 M a^{2}\right) \Delta \sec ^{2} i\right]^{1 / 2}}{\left(r^{3}+r a^{2}+2 M a^{2}\right) \sec i}$

which is essentially the solution for $E$ of $R=0$.

\subsection{Description of pseudo-Newtonian force}

The conditions for a circular orbit are

$$
R=0, \quad \frac{d R}{d r}=0 .
$$

Solving for $E$ and $\lambda_{z}$ from eqn. (17) we obtain

$$
\tilde{E}=\frac{E}{m}=F_{1}(r, a, i) \text { and } \tilde{\lambda}_{z}=\frac{\lambda_{z}}{m}=F_{2}(r, a, i) .
$$


$\tilde{E}$ and $\tilde{\lambda}_{z}$ are specific energy and specific angular momentum parallel to the symmetry axis respectively, and $r$ is the radius of the circular inclined orbit. We do not represent the functions $F_{1}$ and $F_{2}$ explicitly as they are cumbersome. It can be easily verified that $\lambda_{z} \sec i=\lambda$ is the total angular momentum of the orbital plane with inclination angle $i$ and its direction is perpendicular to the plane of the orbit. The relativistic specific angular momentum of the matter is defined by the relation $\ell=-u_{\phi} / u_{t}=\lambda_{z} / E$ (Kozlowski et al. 1978). Following M02 and as standard practice, we can define the Keplerian angular momentum distribution $\lambda_{K}=\ell \sec i$. Therefore, corresponding centrifugal force in the Kerr geometry can be written as

$$
\begin{aligned}
\frac{\lambda_{K}{ }^{2}}{r^{3}} & =\frac{2 M \mathcal{A}^{2} \sec ^{2} i}{\left[a \sqrt{2} \sqrt{M} r^{3 / 2}\{\Delta+2 r(r-M)\}+r \Delta\left\{\left[\mathcal{A}+r^{4}-a^{2}\left(\Delta+r^{2}-3 M r\right)\right] \cos 2 i \sec ^{2} i\right\}^{1 / 2}\right]^{2}} \\
& =F_{G}=F_{K r}
\end{aligned}
$$

where $\mathcal{A}=a^{4}+r^{4}+2 a^{2} r(r-2 M)$. Thus from above, $F_{K r}$ can be identified as the generalized gravitational force of the Kerr black hole at the Keplerian orbit. The subscript $K$ represents a Keplerian orbit and subscript $r$ represents force in the radial direction. The above expression reduces to that of M02 at an inclination angle $i=0$, i.e. particle motion confined to the equatorial plane, and that of PW for $a=i=0$. Thus we can say that above eqn. (19) is the most general form of force corresponding to the PNP for a generalized accretion disk around a rotating black hole. One may be confused with the mathematical form of $F_{K r}$ in eqn. (19) which appears complicated compared to that of PW and thus the PNP approach with $F_{K r}$ appears to be lacking the main motivation and advantage in comparison with full framework of GR. However, one should also remember that mathematical complexity of accretion disk dynamics in full Kerr geometry is beyond comparison with that in much simpler Schwarzschild geometry. Therefore, the apparent complex form of $F_{K r}$ above is nothing compared to the complications exist in the set of actual equations in the Kerr geometry. Therefore, $F_{K r}$ must be acceptable if it exhibits the essential features of the Kerr geometry what we examine in $\S 3$.

The general form of corresponding PNP is

$$
V_{P K}=\int F_{K r} d r
$$

Therefore, $V_{P K}$ is a generalized axisymmetric potential mimicking the geometry of the general Kerr spacetime. The above potential can be attributed to the threedimensional free-fall acceleration of a test particle around a Kerr black hole. In Fig. 2 we show the evolution of the pseudo-Newtonian potential as a function of $r$ and $i$ for extremally rotating black holes. As the unstable region including unbound orbits with small inclination is broader for 
a counter rotating black hole (described in detail in $\S 3$ with Tables 1 and 2), $V_{P K}$ increases and tends to diverge at a larger radius than that of the corotating one. For the corotating case, $V_{P K}$ tends to diverge at a very inner region in the vicinity of the black hole only. Figure 3 describes the corresponding contours of constant $V_{P K}$. It is found that the entire family of contour is shifted towards smaller $r$ for the corotating case compared to the counter rotating one. Above qualitative features are same for other Kerr parameters.

Now we write the Keplerian force components in the radial and vertical direction in cylindrical coordinate system $(\rho, \phi, z)$ from eqn. (19) as

$F_{K \rho}=\frac{2 M \mathcal{A}^{2} \mathcal{B}^{-1 / 2}}{\left[a \sqrt{2 M} \rho^{3 / 2} \mathcal{B}^{1 / 4}\left\{\Delta+2 \rho \mathcal{B}^{1 / 2}\left(\rho \mathcal{B}^{1 / 2}-M\right)\right\}+\Delta \sqrt{\rho^{2}-z^{2}}\left\{\mathcal{A}+\rho^{4} \mathcal{B}^{2}-a^{2}\left(\Delta+\rho^{2} \mathcal{B}-3 M \rho \mathcal{B}^{1 / 2}\right)\right\}^{1 / 2}\right]^{2}}$

and

$F_{K z}=\frac{2 M \mathcal{A}^{2} \mathcal{B}^{-1 / 2} z}{\left[a \sqrt{2 M} \rho^{2} \mathcal{B}^{1 / 4}\left\{\Delta+2 \rho \mathcal{B}^{1 / 2}\left(\rho \mathcal{B}^{1 / 2}-M\right)\right\}+\Delta \rho^{1 / 2} \sqrt{\rho^{2}-z^{2}}\left\{\mathcal{A}+\rho^{4} \mathcal{B}^{2}-a^{2}\left(\Delta+\rho^{2} \mathcal{B}-3 M \rho \mathcal{B}^{1 / 2}\right)\right\}^{1 / 2}\right]^{2}}$

respectively, where $\mathcal{B}=1+z^{2} / \rho^{2}, \Delta=\rho^{2} \mathcal{B}-2 M \rho \mathcal{B}^{1 / 2}+a^{2}, \mathcal{A}=a^{4}+\rho^{4} \mathcal{B}^{2}+2 a^{2} \rho \mathcal{B}^{1 / 2}\left(\rho \mathcal{B}^{1 / 2}-\right.$ $2 M) . \quad F_{K \rho}$ and $F_{K z}$ are the components of force corresponding to the pseudo-Newtonian vector potential components in cylindrical geometry.

Once we have the pseudo-Newtonian potential (and force), we examine the corresponding density distribution $d$ from the Poisson's equation

$$
-4 \pi d=\frac{1}{\rho} \frac{\partial}{\partial \rho}\left(\rho F_{K \rho}\right)+\frac{\partial}{\partial z} F_{K z}
$$

shown in Fig. 4 for extremally rotating black holes. It is found that the hypothetical density distribution $d$ is Gaussian along $z$ axis for both corotating and counterrotating black holes. Like $V_{P K}, d$ increases much faster at an outer radius for the counterrotating black hole than the corotating one. $d$ for the corotating case increases significantly only at very close to the black hole event horizon.

\section{Comparison of the essential features for the Kerr geometry and pseudo-Kerr}

To check the validity of the potential derived in the preceding section, we follow the procedure considered in M02 and Mukhopadhyay \& Misra (2003). We make a comparison 
to show how far the potential $V_{P K}$ reproduces the values of $r_{s}, r_{b}$ and $E_{s}$ as that in Kerr geometry. We do not repeat the corresponding equations to calculate the parameters in Kerr geometry as they are established well in literature (see e.g. Shapiro \& Teukolsky). However, for completeness, we show them for the pseudo-Kerr case. Stability of an orbit requires $(\mathrm{PW})$

$$
\frac{d}{d r}\left(\lambda_{K}\right) \geq 0
$$

Therefore, we obtain marginally stable orbit $\left(r_{s}\right)$ by solving eqn. (24) for $r$ with "equals to" sign.

The energy of a particle in the Kerr geometry according to $V_{P K}$ is given by

$$
E=\frac{r}{2} \frac{d V_{P K}}{d r}+V_{P K}
$$

Therefore marginally bound orbit $\left(r_{b}\right)$ can be accounted for by solving the equation

$$
\left.E\right|_{r_{b}}=0
$$

for $r_{b}$. In addition, once we know $r_{s}$, we can compute $\left.E\right|_{r_{s}}=E_{S}$, the energy at last stable circular orbit. We enlist the comparison of $r_{s}, r_{b}$ and $E_{s}$ between the Kerr and pseudo-Kerr geometry with percentage error for a few inclination parameters $i$ in the Tables 1,2,3. We present the values of above parameters upto the orbital inclination $i=30^{\circ}$, as we believe that realistically the disk thickness does not exceed beyond the height corresponding to inclination parameter $i \sim 30^{\circ}$. It is exciting to note that $r_{s}$ computed from pseudo-Kerr geometry exactly matches to that in Kerr geometry for all values of $a$ and $i$. To best of our knowledge, none of the existing pseudo-Newtonian potentials proposed for threedimensional Kerr geometry reproduces $r_{s}$ exactly. Table 1 shows that values of $r_{b}$ obtained from $V_{P K}$ match with that from exact Kerr geometry in very good agreement; with a maximum error $\sim 7 \%$ at high inclination angle $30^{\circ}$. With the increase of $a$ upto certain value, error increases to a maximum (obtained in the range $0.998 \geq a \geq 0.9$ ) and then decreases to 0 at $a=1$. It is to be noted that values of $r_{s}$ and $r_{b}$ are independent of $i$ at $a=1$ which coincide with the event horizon as the case for $i=0$. The energy of an accreting particle at marginally stable orbit is also being reproduced by our PNP in very good agreement with that in exact Kerr geometry for $i \lesssim 20^{\circ}$, as understood from Table 2 . Although the error increases with the increase of $i$ beyond $20^{\circ}$, except for $a=1$ which is a special case, it is still within $20 \%$ for any realistic disk. For counter-rotating black holes the errors are less than that for co-rotating ones. However, it is to be noted that for very high inclination angle this trend reverses in $E_{s}$ as shown in Fig. 5 and Table 2. In both the cases of Kerr and pseudo-Kerr geometry, the orbits recede with the increase of inclination parameter $i$ for prograde black holes, and 
for retrograde black holes this dynamics is opposite. We compare the variation of specific energy for circular Keplerian orbit with the radial distance in Fig. 5 computed for exact

Kerr geometry and our PNP for both prograde and retrograde holes at orbital inclination $10^{\circ}$ and $30^{\circ}$.

The pseudo-Newtonian potential described by Semerák \& Karas (1999), while tries to capture frame-dragging effects, can not reproduce other general relativistic properties e.g. radii of marginally stable and bound orbit, energy at marginally stable orbit. These are very important for the purpose of accretion disk producing jet, expected to be launched from inner edge of the disk. On the other hand, our potential is derived directly from the spacetime metric, more precisely from the effective potential of the threedimensional Kerr geometry given by eqns. (15) and (16). Either of the equations inherently carries information of frame-dragging effects with the term proportional to $a \lambda_{z}$. Therefore, the frame-dragging effects are expected to be guaranteed by our potential. While the ad-hoc potential given by Chakrabarti \& Mondal (2006) tries to mimic frame-dragging as well as inner disk properties both, it is not valid for entire parameter regime. It also does not reproduce the radii of marginally stable orbit exactly as ours does. While their potential is a function of $r$ and $\theta$ both, they discuss most of the results at equatorial plane $(\theta=\pi / 2)$ and thus it is not clear how effective their potential is in a real system. Therefore, our potential is expected to be most selfcontained and useful to study generalized accretion disk including jet, particularly for the hydrodynamical/magnetohydrodynamical analysis.

Thus, for a realistic accretion disk, our PNP, although approximate in nature, should reproduce the essential features of the spacetime in very good agreement. The potential is effectively useful to study the detailed hydrodynamics of the realistic disk with the inclusion of vertical outflow using Newtonian MHD equations, especially in the inner region, where general relativistic effects are very important. Thus the potential satisfies all the criteria to be claimed as good pseudo-Newtonian vector potential for a general Kerr metric.

\section{Discussion}

In this paper, we have prescribed a most generalized gravitational potential, pseudoNewtonian vector potential, for the modeling of thick accretion disks around rotating black holes. As this potential is derived from the spacetime metric directly, it naturally carries several essential general relativistic properties. Our potential is valid for co-rotating as well as counter-rotating black holes with all realistic values of orbital inclination. The inherent nature of reproducing exact values of $r_{s}$ for the entire regime of Kerr parameter and for all inclination angle by our PNP makes it an efficient choice. The prescribed PNP also 
reproduces $r_{b}$ in exact Kerr geometry with a maximum error $\sim 7 \%$ for a high inclination $\sim 30^{\circ}$. However, it can be noted that the potential may not be a good approximation for the angular and epicyclic frequencies for less than 20 Schwarzschild radii.

The full scale disk dynamics is very complicated and there is no virtue to assume that the accretion disk around black holes is fully Keplerian in nature. Moreover, the vertical outflow and wind in the form of relativistic jet, which are believed to be present almost in all quasars and microquasars, are predicted to be emanating from a very inner region of a puffed up disk or from a coronal transition region (Liu et al. 1999; Meyer et al. 2000). In addition, various inner fluid dynamical properties and also the powering of jet depend on spin parameter of the black hole. Thus evidently the realistic accretion physics (especially the accretion dynamics) around black holes can be studied with our prescribed axisymmetric generalized PNP, capturing the essential physics of GR.

The general relativistic study of full scale dynamics of a realistic accretion disk for a highly turbulent viscous medium with the inclusion of jet and magnetic field is beyond the scope with the present physical and mathematical tools available to us. Most of the numerical studies of general relativistic flows have been performed with simplifying assumptions and remained confine to certain regions. Such simulations do not explicitly disclose the underlying microphysics of the structure and dynamics of disks and jets. In order to avoid complexities in full GR frameworks and with a notion to understand the underlying physics of the symbiotic connection of disk and jet, our PNP is highly recommended. Even if the results using our PNP deviate quantitatively by a few percent from that of the actual Kerr geometry, the accurate physical interpretation of the activities around black holes is possible. Our generalized PNP can be used to perform quasi-analytical studies and time-dependent simulations of accretion and wind and related physics like that done by Chan et al. (2005, 2007), Lipunov \& Gorbovskoy (2007), Shafee et al. (2007) for more realistic situations.

Next, out of several plans, we would like to use our very approach to understand the physics of the symbiotic connection of disk and jet around black holes which, in the astrophysical context, is called by jet-disk symbiosis. In this context, one can extend the qualitative picture of the jet-disk symbiosis developed earlier (Falcke \& Biermann 1995, Donea \& Biermann 1996) to a full scale threedimensional MHD in the Newtonian framework with the inclusion of our PNP. The structure of the disk is highly modified in presence of the jet. Thus, we plan to describe the disk including all the above mentioned effects in a realistic situation, and to develop a tool to test with observation, both of the disk and the jet (from radio to TEV photons).

The authors thank the referee for his/her very useful and constructive suggestions which 
helped to improve the presentation of the paper. They also thank Paul J. Wiita for his comments in preparing the final version of the manuscript.

\section{REFERENCES}

Artemova, I. V., Björnsson, G., \& Novikov, I. D. 1996, ApJ, 461, 565.

Bardeen, J. M., Press, W. H., \& Teukolsky, S. A. 1972, ApJ, 178, 347.

Bondi, H. 1952, MNRAS, 112, 195.

Carter, B. 1968, Phys. Rev, 174, 1559.

Chakrabarti, S. K., \& Khanna, R. 1992, MNRAS, 256, 300.

Chakrabarti, S. K., \& Mondal, S. 2006, MNRAS, 369, 976.

Chan, C., Psaltis, D., \& Özel, F. 2005, ApJ, 628, 353.

Chan, C., Psaltis, D., \& Özel, F. 2007, ApJ Submitted, arXiv:astro-ph/0612742.

Chandrasekhar, S. 1983, The Mathematical theory of black holes. (Clarendon press. Oxford)

Dadhich, N. 1985, in A Random Walk in Relativity and Cosmology, eds. N. Dadhich \& C. V. Vishveshwara (New Delhi: Wiley Eastern), 72.

Donea, A.-C., \& Biermann, P. 1996, A\&A, 316, 43.

Falcke, H., \& Biermann, P. 1995, A\&A, 293, 665.

Genzel, R., Schödel, T. Ott., Eckart, A., Alexander, T., Lacombe, F., Rouan, D., \& Aschenbach, B. 2003, Nature, 425, 934.

Ghosh, S. 2004, A\&A, 418, 795.

Glampedakis, K., Hughes, S. A., Kennefick, D. 2002, Phys. Rev. D, 66, 064005.

Hughes, S. A. 2000, Phys. Rev. D, 61, 084004.

Ivanov, R. I., Prodanov, E. M., 2005, Phys. Lett. B, 611, 34

Iwasawa, K., Fabian, A. C., Reynolds, C. S., Nandra, K., Otani, C., Inoue, H., Hayashida, K., Brandt, W. N., Dotani, T., Kunieda, H., Matsuoka, M., \& Tanaka, Y. 1996, MNRAS, 282, 1038. 
Kozlowski, M., Jaroszyński, M., \& Abramowicz, M. A. 1978, A\&A, 63, 209.

Lens, J., \& Thirring, H., 1918, Phys. Z. 19, 156.

Lipunov, V., \& Gorbovskoy, E. 2007, ApJ Submitted, arXiv:0705.1648.

Liu, B. F., Yuan, W., Meyer, F., Meyer-Hofmeister, E., \& Xie, G. Z. 1999, ApJ, 527, L17.

Lovas, T. 1998, IJMPD, 7, 471.

McClintock, J. E., Shafee, R., Narayan, R., Remillard, R. A., Davis, S. W., \& Li, L.-X. 2006, ApJ, 652, 518.

Meyer, F., Liu, B. F., \& Meyer-Hofmeister, E. 2000, A\&A, 361, 175.

Michel, F. Curtis. 1972, Ap\&SS, 15, 153.

Miller, J. M. et al. 2004, ApJ, 601, 450.

Miniutti, G., Fabian, A. C., \& Miller, J. M. 2004, MNRAS, 351, 466.

Mukhopadhyay, B. 2002, ApJ, 581, 427; M02.

Mukhopadhyay, B., Misra, R. 2003, ApJ, 582, 347.

Mukhopadhyay, B. 2003, ApJ, 586, 1268.

Novikov, I. D., \& Thorne, K. S. 1973, in Black Holes, Les Houches 1972 (France), ed. B. \& C. DeWitt (New York: Gordon \& Breach), 343.

Nowak, A. M., \& Wagoner, R. V. 1991, ApJ, 378, 656.

Paczyński, B., \& Wiita, P. J. 1980, A\&A, 88, 23; PW.

Semerák, O., \& Karas, V. 1999, A\&A, 343, 325.

Shafee, R., \& Narayan, R., \& McClintock, J. E. 2007, ApJ Submitted, arXiv:0705.2244.

Shakura, N., \& Sunyaev, R. 1973, A\&A, 24, 337.

Shapiro, S. L. 1996, Phys. Rev. L, 77, 4487.

Shapiro, S. L., \& Teukolsky, S. A. 1983, Black Holes, White Dwarfs, and Neutron Stars: Physics of Compact Objects (New York: John Wiley \& Sons.).

Ryan, F. D. 1995, Phys. Rev. D, 52, 315. 
Wilkins, D. C. 1972, Phys. Rev. D, 5, 814.

Wilms, J., Reynolds, C. S., Begelman, M. C., Reeves, J., Molendi, S., Staubert, R., \& Kendziorra, E. 2001, MNRAS, 328, 27. 
Table 1: Values of $r_{b}$

\begin{tabular}{|c|c|c|c|c|c|c|c|c|c|c|c|c|}
\hline \multirow{3}{*}{$a$} & \multicolumn{3}{|c|}{$\mathrm{K}$} & \multicolumn{3}{|c|}{$\mathrm{K}$} & \multicolumn{3}{|c|}{$\mathrm{K}$} & \multicolumn{3}{|c|}{$\mathrm{K}$} \\
\hline & $i$ & & Er & $i$ & & Er & $i$ & & Er & $i$ & & Er \\
\hline & \multicolumn{3}{|c|}{ PK } & \multicolumn{3}{|c|}{ PK } & \multicolumn{3}{|c|}{ PK } & \multicolumn{3}{|c|}{ PK } \\
\hline \multirow{3}{*}{1.0} & \multirow{3}{*}{0} & 1.0 & \multirow{3}{*}{0.0} & \multirow{3}{*}{10} & 1.0 & \multirow{3}{*}{0.0} & \multicolumn{3}{|c|}{1.0} & \multirow{2}{*}{\multicolumn{2}{|c|}{1.0}} & \multirow{3}{*}{0.0} \\
\hline & & & & & & & 20 & & 0.0 & & & \\
\hline & & 1.0 & & & 1.0 & & & 1.0 & & & 1.0 & \\
\hline \multirow{3}{*}{0.998} & \multirow{3}{*}{0} & 1.0914 & & & 1.0942 & & & 1.1048 & & & 1.1369 & \\
\hline & & & 5.0119 & 10 & & 5.1088 & 20 & & 5.4671 & 30 & & 6.6233 \\
\hline & & 1.0367 & & & 1.0383 & & & 1.0444 & & & 1.0616 & \\
\hline & & 1.7325 & & & 1.7459 & & & 1.7890 & & & 1.8706 & \\
\hline 0.9 & 0 & & 4.6118 & 10 & & 4.5535 & 20 & & 4.3823 & 30 & & 4.0468 \\
\hline & & 1.6526 & & & 1.6664 & & & 1.7106 & & & 1.7949 & \\
\hline & & 2.3955 & & & 2.4106 & & & 2.457 & & & 2.5369 & \\
\hline 0.7 & 0 & & 2.5924 & 10 & & 2.5429 & 20 & & 2.4217 & 30 & & 2.1838 \\
\hline & & 2.3334 & & & 2.3493 & & & 2.3975 & & & 2.4815 & \\
\hline & & 2.9142 & & & 2.9268 & & & 2.9648 & & & 3.0281 & \\
\hline 0.5 & 0 & & 1.5064 & 10 & & 1.4760 & 20 & & 1.3964 & 30 & & 1.2615 \\
\hline & & 2.8703 & & & 2.8836 & & & 2.9234 & & & 2.9899 & \\
\hline & & 3.3733 & & & 3.3816 & & & 3.4064 & & & 3.4473 & \\
\hline 0.3 & 0 & & 0.7826 & 10 & & 0.7541 & 20 & & 0.7134 & 30 & & 0.6469 \\
\hline & & 3.3469 & & & 3.3561 & & & 3.3821 & & & 3.4250 & \\
\hline & & 3.7974 & & & 3.8003 & & & 3.8091 & & & 3.8235 & \\
\hline 0.1 & 0 & & 0.2317 & 10 & & 0.2079 & 20 & & 0.1969 & 30 & & 0.1805 \\
\hline & & 3.7886 & & & 3.7924 & & & 3.8016 & & & 3.8166 & \\
\hline & & 4 & & & 4 & & & 4 & & & 4 & \\
\hline 0 & 0 & & 0 & 10 & & 0 & 20 & & 0 & 30 & & 0 \\
\hline & & 4 & & & 4 & & & 4 & & & 4 & \\
\hline & & 4.1976 & & & 4.1945 & & & 4.1853 & & & 4.1702 & \\
\hline-0.1 & 0 & & 0.2311 & 10 & & 0.2265 & 20 & & 0.2150 & 30 & & 0.2038 \\
\hline & & 4.2073 & & & 4.2040 & & & 4.1943 & & & 4.1787 & \\
\hline & & 4.5804 & & & 4.5706 & & & 4.5418 & & & 4.4949 & \\
\hline-0.3 & 0 & & 0.5960 & 10 & & 0.5907 & 20 & & 0.5681 & 30 & & 0.5273 \\
\hline & & 4.6077 & & & 4.5976 & & & 4.5676 & & & 4.5186 & \\
\hline & & 4.9495 & & & 4.9328 & & & 4.8831 & & & 4.8022 & \\
\hline-0.5 & 0 & & 0.9072 & 10 & & 0.8981 & 20 & & 0.8683 & 30 & & 0.8142 \\
\hline & & 4.9944 & & & 4.9771 & & & 4.9255 & & & 4.8413 & \\
\hline & & 5.3077 & & & 5.2836 & & & 5.2121 & & & 5.0953 & \\
\hline-0.7 & 0 & & 1.1775 & 10 & & 1.1659 & 20 & & 1.1320 & 30 & & 1.0716 \\
\hline & & 5.3702 & & & 5.3452 & & & 5.2711 & & & 5.1499 & \\
\hline & & 5.6568 & & & 5.6251 & & & 5.5308 & & & 5.3765 & \\
\hline-0.9 & 0 & & 1.4160 & 10 & & 1.4044 & 20 & & 1.3687 & 30 & & 1.3038 \\
\hline & & 5.7369 & & & 5.7041 & & & 5.6065 & & & 5.4466 & \\
\hline & & 5.8250 & & & 5.7895 & & & 5.6837 & & & 5.5104 & \\
\hline-0.998 & 0 & & 1.5227 & 10 & & 1.5096 & 20 & & 1.4762 & 30 & & 1.4137 \\
\hline & & 5.9137 & & & 5.8769 & & & 5.7676 & & & 5.5883 & \\
\hline & & 5.8284 & & & 5.7928 & & & 5.6868 & & & 5.5131 & \\
\hline-1.0 & 0 & & 1.4927 & 10 & & 1.4794 & 20 & & 1.4419 & 30 & & 1.3731 \\
\hline & & 5.9154 & & & 5.8785 & & & 5.7688 & & & 5.5888 & \\
\hline
\end{tabular}

Note. - Percentage error=Er. Inclination angle $i$ expressed in degrees, $\mathrm{K}$ and PK denote Kerr and pseudo-Newtonian result respectively. 
Table 2: Values of $E_{s}$

\begin{tabular}{|c|c|c|c|c|c|c|c|c|c|c|c|c|}
\hline \multirow{3}{*}{$a$} & \multirow{3}{*}{$i$} & \multicolumn{2}{|l|}{$\overline{\bar{K}}$} & \multicolumn{3}{|c|}{$\overline{\mathrm{K}}$} & \multicolumn{3}{|c|}{$\bar{K}$} & \multicolumn{3}{|c|}{$\bar{K}$} \\
\hline & & & Er & $i$ & & Er & $i$ & & Er & $i$ & & Er \\
\hline & & PK & & & PK & & & PK & & & PK & \\
\hline \multirow{3}{*}{1.0} & \multirow{3}{*}{0} & 0.0 & \multirow{3}{*}{0.0} & \multirow{3}{*}{10} & 0.0 & & \multirow{3}{*}{20} & 0.0 & & & 0.0 & \multirow{3}{*}{0.0} \\
\hline & & & & & & 0.0 & & & 0.0 & 30 & & \\
\hline & & 0.0 & & & 0.0 & & & 0.0 & & & 0.0 & \\
\hline \multirow{3}{*}{0.998} & \multirow{3}{*}{0} & -0.3210 & \multirow{3}{*}{10.0623} & \multirow{3}{*}{10} & -0.3118 & & \multirow{3}{*}{20} & -0.2816 & & & -0.2264 & \multirow{3}{*}{15.7243} \\
\hline & & & & & & 6.8954 & & & 2.1307 & 30 & & \\
\hline & & -0.3533 & & & -0.3333 & & & -0.2756 & & & -0.1908 & \\
\hline \multirow{3}{*}{0.9} & & -0.1558 & & & -0.1530 & & & -0.1450 & & & -0.1327 & \\
\hline & 0 & & 12.1951 & 10 & & 8.8235 & 20 & & 0.8966 & 30 & & 15.9005 \\
\hline & & -0.1748 & & & -0.1665 & & & -0.1437 & & & -0.1116 & \\
\hline & & -0.1036 & & & -0.1026 & & & -0.0995 & & & -0.0949 & \\
\hline 0.7 & 0 & & 10.8108 & 10 & & 7.4074 & 20 & & 2.1106 & 30 & & 15.9652 \\
\hline & & -0.1148 & & & -0.1102 & & & -0.0974 & & & -0.0788 & \\
\hline & & -0.0821 & & & -0.0816 & & & -0.0802 & & & -0.0780 & \\
\hline 0.5 & 0 & & 9.9757 & 10 & & 6.7402 & 20 & & 2.8678 & 30 & & 17.5641 \\
\hline & & -0.0904 & & & -0.0871 & & & -0.0779 & & & -0.0643 & \\
\hline & & -0.0694 & & & -0.0691 & & & -0.0685 & & & -0.0675 & \\
\hline 0.3 & 0 & & 9.5101 & 10 & & 6.3676 & 20 & & 3.2117 & 30 & & 17.7778 \\
\hline & & -0.0760 & & & -0.0735 & & & -0.0663 & & & -0.0555 & \\
\hline & & -0.0606 & & & -0.0606 & & & -0.0604 & & & -0.0602 & \\
\hline 0.1 & 0 & & 9.2409 & 10 & & 5.9406 & 20 & & 3.4768 & 30 & & 18.1063 \\
\hline & & -0.0662 & & & -0.0642 & & & -0.0583 & & & -0.0493 & \\
\hline & & -0.0572 & & & -0.0572 & & & -0.0572 & & & -0.0572 & \\
\hline 0 & 0 & & 9.0909 & 10 & & 5.9441 & 20 & & 3.4965 & 30 & & 18.1818 \\
\hline & & -0.0624 & & & -0.0606 & & & -0.0552 & & & -0.0468 & \\
\hline & & -0.0542 & & & -0.0542 & & & -0.0544 & & & -0.0546 & \\
\hline-0.1 & 0 & & 9.0406 & 10 & & 5.7196 & 20 & & 3.8603 & 30 & & 18.3150 \\
\hline & & -0.0591 & & & -0.0573 & & & -0.0523 & & & -0.0446 & \\
\hline & & -0.0492 & & & -0.0493 & & & -0.0496 & & & -0.0502 & \\
\hline-0.3 & 0 & & 8.7398 & 10 & & 5.4767 & 20 & & 3.8306 & 30 & & 18.5259 \\
\hline & & -0.0535 & & & -0.0520 & & & -0.0477 & & & -0.0409 & \\
\hline & & -0.0451 & & & -0.0453 & & & -0.0458 & & & -0.0465 & \\
\hline-0.5 & 0 & & 8.8647 & 10 & & 5.2980 & 20 & & 4.1485 & 30 & & 18.4946 \\
\hline & & -0.0490 & & & -0.0477 & & & -0.0439 & & & -0.0379 & \\
\hline & & -0.0418 & & & -0.0420 & & & -0.0426 & & & -0.0435 & \\
\hline-0.7 & 0 & & 8.3732 & 10 & & 5.2381 & 20 & & 4.2254 & 30 & & 18.6207 \\
\hline & & -0.0453 & & & -0.0442 & & & -0.0408 & & & -0.0354 & \\
\hline & & -0.0390 & & & -0.0392 & & & -0.0398 & & & -0.0409 & \\
\hline-0.9 & 0 & & 8.2051 & 10 & & 5.1020 & 20 & & 4.2714 & 30 & & 18.5819 \\
\hline & & -0.0422 & & & -0.0412 & & & -0.0381 & & & -0.0333 & \\
\hline & & -0.0378 & & & -0.0380 & & & -0.0387 & & & -0.0398 & \\
\hline-0.998 & 0 & & 8.4656 & 10 & & 5.2632 & 20 & & 3.8760 & 30 & & 17.8392 \\
\hline & & -0.0410 & & & -0.0400 & & & -0.0372 & & & -0.0327 & \\
\hline & & -0.0377 & & & -0.0380 & & & -0.0386 & & & -0.0398 & \\
\hline-1.0 & 0 & & 8.7533 & 10 & & 5.2632 & 20 & & 3.8860 & 30 & & 17.8392 \\
\hline & & -0.0410 & & & -0.0400 & & & -0.0371 & & & -0.0327 & \\
\hline
\end{tabular}

Note. - Percentage error=Er. Inclination angle $i$ expressed in degrees, $\mathrm{K}$ and PK denote Kerr and pseudo-Newtonian result respectively. 


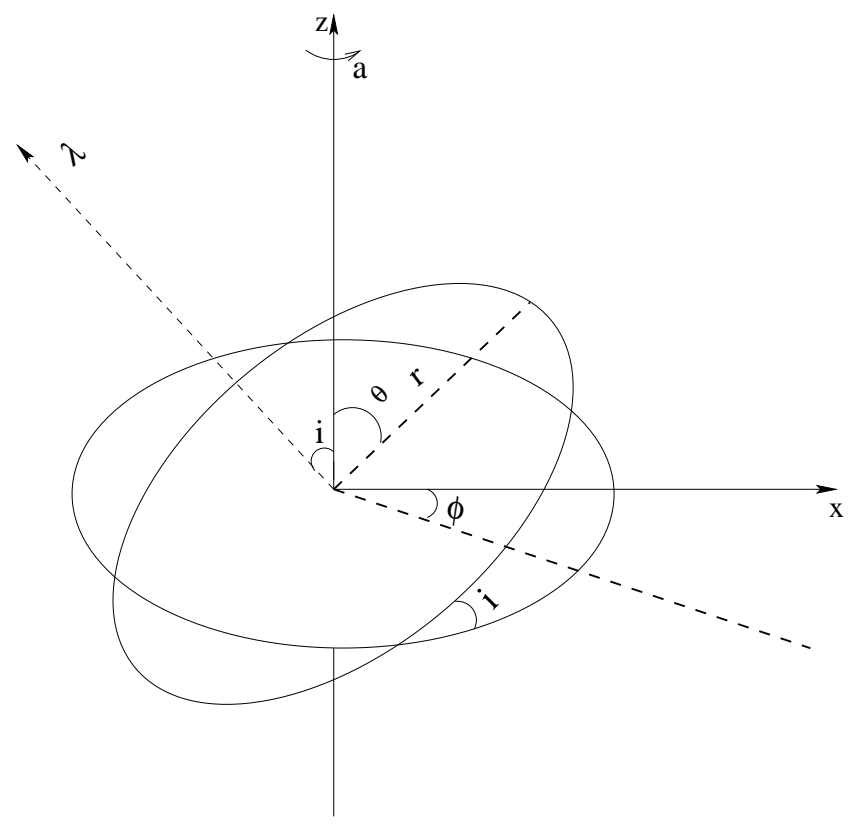

Fig. 1. - Schematic diagram showing how an orbit of the accreting particle inclines to the equatorial plane. The orbit, inclined at an angle $i$, represents a generalized accretion. $\lambda$ represents the total angular momentum of the orbiting particle, $a$ is the spin parameter of the black hole and $z$ axis is the symmetry axis of the system. 

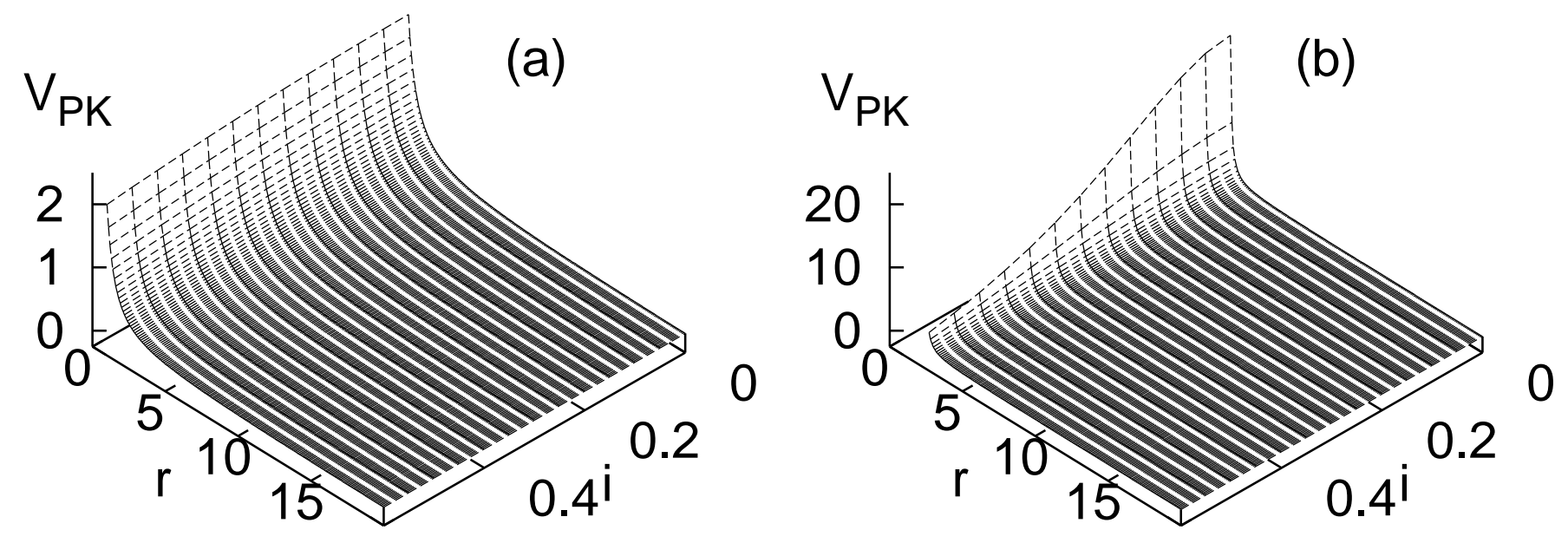

Fig. 2.- Variation of generalized pseudo-Newtonian potential $V_{P K}$ as a function of $r$ (in units of $G M / c^{2}$ ) and $i$ (in radian) for (a) $a=1$, (b) $a=-1$. 

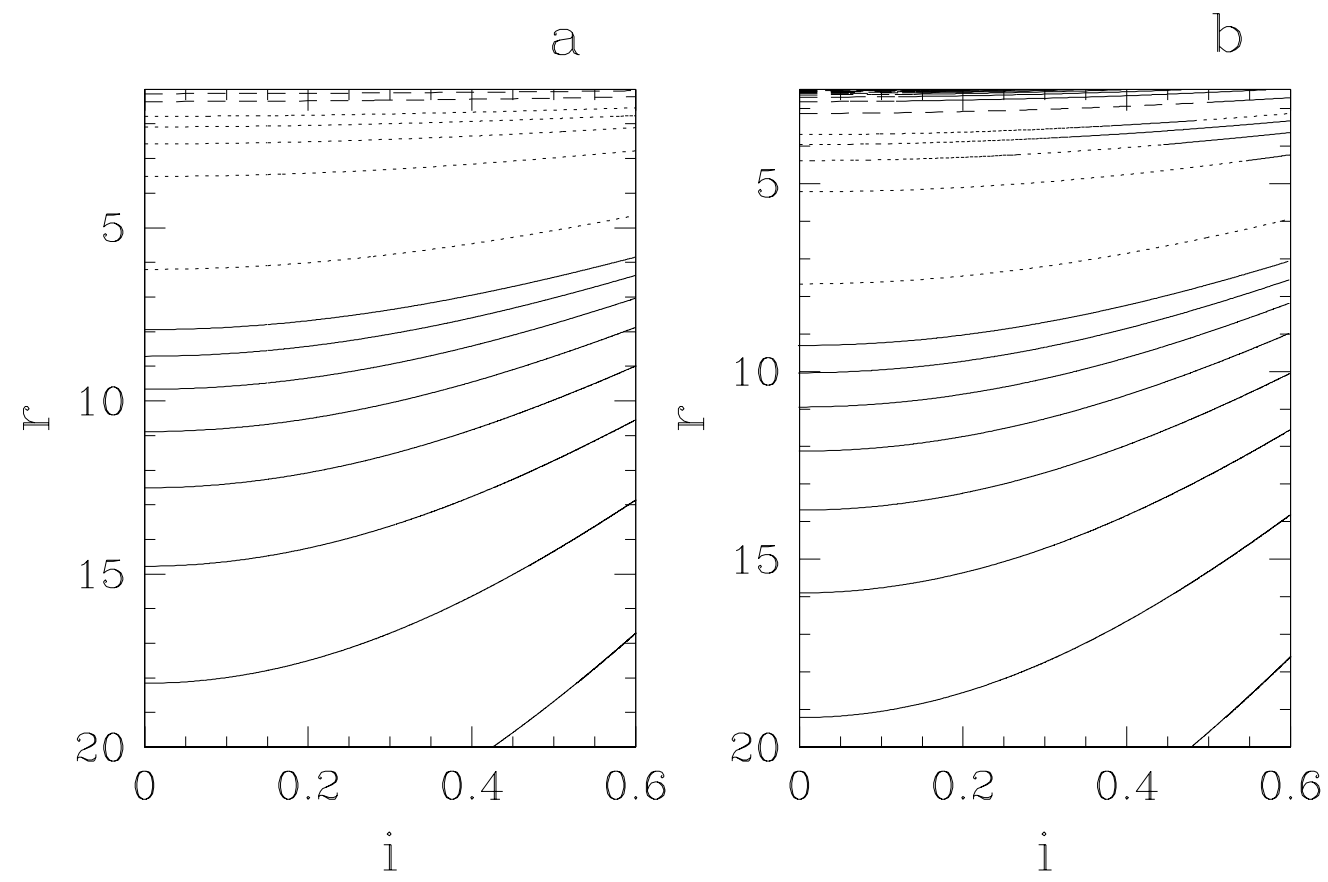

Fig. 3.- Contours of constant $V_{P K}$ for (a) $a=1$, when solid lines correspond to $V_{P K}=$ $0.045,0.06,0.075, \ldots \ldots, 0.15$, dotted lines to $0.2,0.4,0.6, \ldots \ldots, 1$, and dashed lines to $1.5,2$, (b) $a=-1$, when solid lines correspond to $V_{P K}=0.045,0.06,0.075, \ldots \ldots, 0.15$, dotted lines to $0.2,0.4,0.6, \ldots \ldots, 1$, and dashed lines to $2,4,6, \ldots \ldots, 10$. 
(a)

(b)
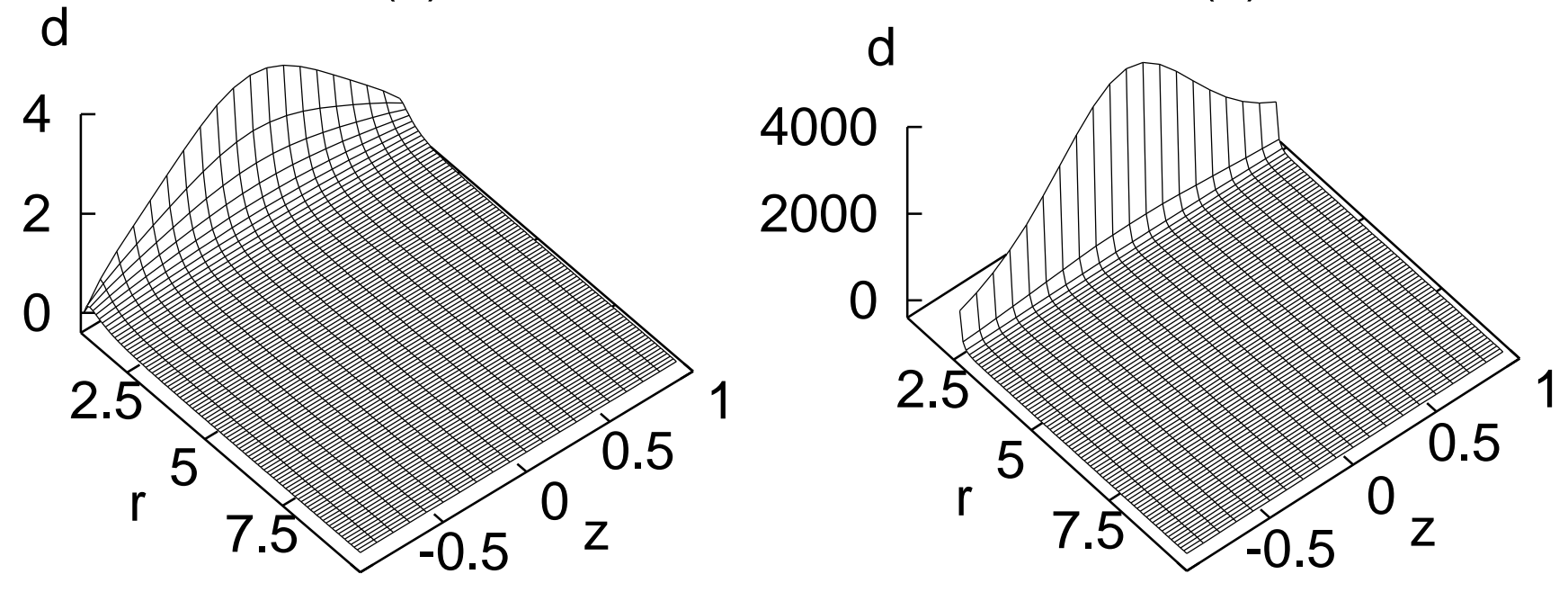

Fig. 4.- Variation of density distribution generating $V_{P K}$ as a function of $r$ and $z$ (in units of $G M / c^{2}$ ) for (a) $a=1$, (b) $a=-1$. 

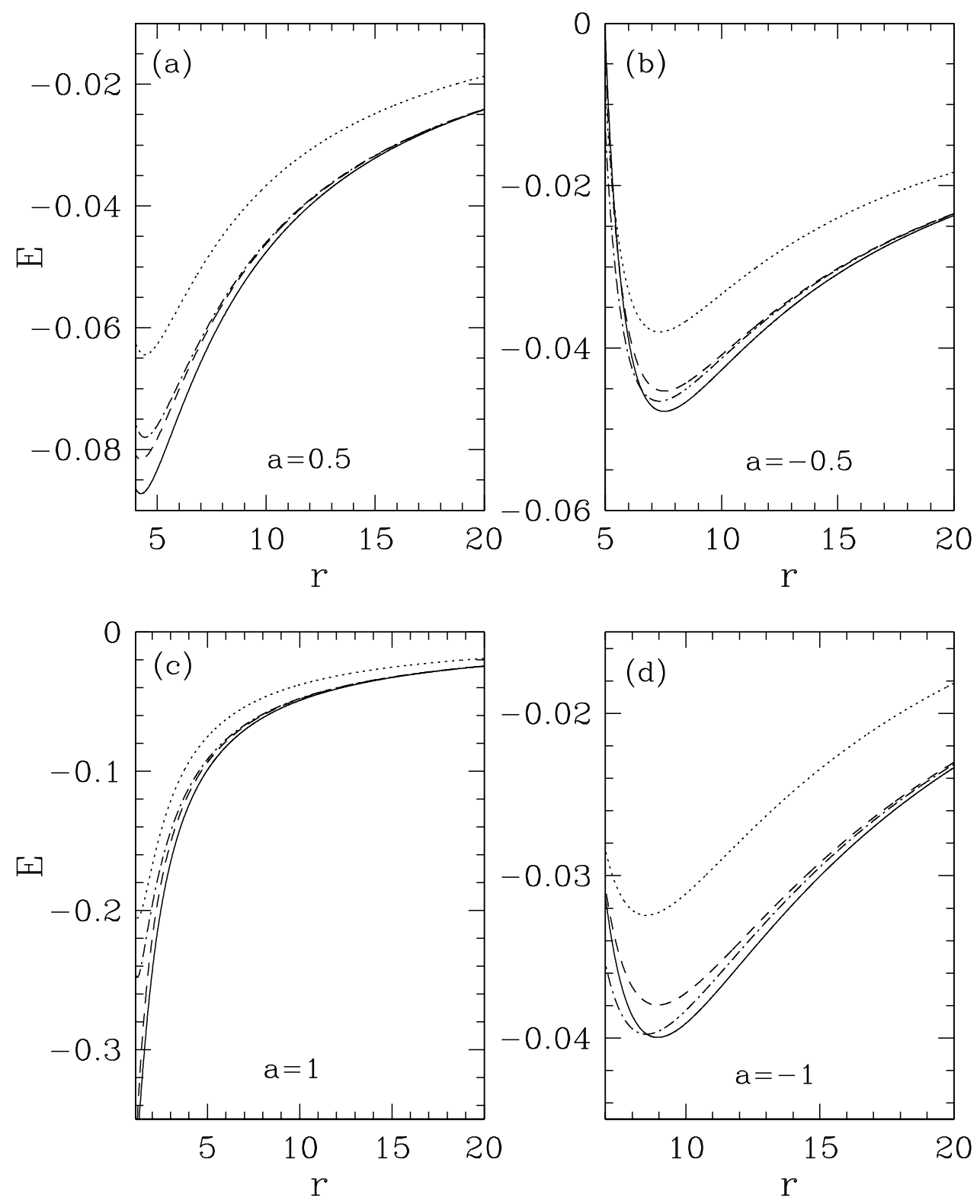

Fig. 5.- Variation of specific energy for circular orbits as a function of radial coordinate at various Kerr parameters. The solid and dotted curves are for our pseudo-Newtonian potential with $i=10^{\circ}$ and $30^{\circ}$ respectively and the dashed and dot-dashed curves are for Kerr geometry with $i=10^{\circ}$ and $30^{\circ}$ respectively at each panel. 